WORLD SCIENTIFIC SERIES ON DIRECTIONS IN CONDENSED MATTER PHYSICS

Vol. 1 : Directions in Condensed Matter Physics

Memorial Volume in Honor of Shang-keng Ma

edited by G. Grinstein \& G. Mazenko

Forthcoming:

Vol. 2 : Ionic Solids at High Temperatures

edited by $A$. Stoneham

Vol. 4 : Directions in Chaos (Vol. 2)

edited by B. - L. Hao

Vol. 5 : Defect Processes Induced by Electronic Excitation in Insulators edited by N. Itoh

Vol. 6 : Spin Glasses and Biology

edited by D. Stein

Vol. 7 : Spin Glasses

edited by J. Hertz

Vol. 8 : Classical Wave Localisation

edited by P. Sheng 
World Scientific Series on Directions in Condensed Matter Physics - Vol. 3

\section{DIRECTIONS IN CHAOS}

Volume 1

\section{Edited by \\ Hao Bai-lin}


Published by

World Scientific Publishing Co. Pte. Ltd.

P.O. Box 128, Farrer Road, Singapore 9128

U.S.A. office: World Scientific Publishing Co., Inc.

687 Hartwell Street, Teaneck NJ 07666, USA

Library of Congress Cataloging-in-Publication data is available.

\section{DIRECTIONS IN CHAOS (Vol. 1)}

Copyright $\odot 1987$ by World Scientific Publishing Co Pte Ltd.

All rights reserved. This book, or parts thereof, may not be reproduced in any form or by any means, electronic or mechanical, including photocopying, recording or any information storage and retrieval system now known or to be invented, without written permission from the Publisher.

\section{ISBN 9971-50-359-X \\ $9971-50-360-3 \mathrm{pbk}$}

Printed in Singapore by Fong and Sons Printers Pte. Ltd. 


\section{FOREWORD}

The idea of publishing a review volume on current topics in the study of chaotic phenomena occurred after discussions with Dr. K. K. Phua, editor-in-Chief of WSPC. The volume is to be a forum for researchers working on nonlinear dynamics as well as for graduate students entering this field. As no severe limitations on the style and length of the reviews have been imposed, the authors are quite free in writing their contributions, and, at the same time, are responsible for their own point of view. In this way we hope to bridge an existing gap between review journals and periodicals for original papers, as no journal can publish several reviews on chaos in one year and some topics worthy to be reviewed for the "chaotic" community may seem too specific for a wider audience.

Since the first response to the request to contribute to this volume was quite enthusiastic, I had to split the planned volume into two parts. As Dr. J. Ford's article on general aspects of classical chaos has released me from the duty to write a longer Foreword, I shall confine myself to a very brief summary of the other papers in the first part.

In a sense, the most important contribution of physicists to the study of chaos consists in introducing the idea and technique of renormalization group. The paper of L. Reichl and W.-M. Zheng reviews the renormalization group approach to the occurrence of stochasticity in classical conservative systems, and K. L. Liu and K. Young's paper deals with the generalization of the celebrated FeigenbaumCvitanovic renormalization group equation for one-dimensional mappings.

The three following papers treat one or another aspect of experimental chaos. The measurement of various dimensions has become a major means to characterize chaotic attractors and is being used by researchers ranging from physics to physiology and economy. Dr. Mayer-Kress reviews the recent development and problems in applying dimensional algorithms to experimental data. The review by L. R. Tredicce and L.M. Narducci deals with truly optical chaos in laser systems, as compared to chaos observed in optical bistable devices where the characteristic frequency of attractors falls far below that of optics. J.-M. Yuan's review on classical, semiclassical and quantum study of the dissipative Morse oscillator provides a more realistic model to understand the manifestation of chaos in multiphoton excitation and dissociation of molecules.

The apparently lack of complete period-doubling sequences in higher dimensional volume-preserving mappings has been a puzzle for some time. The resolution of this subtle problem is reviewed in the first part of J.-M. Mao and Bambi Hu's 
paper. The second part of their paper discusses the quasiperiodic transition to chaos in systems with three incommensurable frequencies, summarizing partially a long-circulated and yet unpublished preprint of J. Guckenheimer, Bambi Hu and J. Rudnick.

We close this volume with a review by J. Crutchfield and K. Kaneko on spatialtemporal chaos. This is an inevitable development towards the understanding of real turbulence after enough knowledge has been gained on the time evolution of simple nonlinear systems and competition of spatial periodicities. This direction leads to a hierarchy of models.

June 30,1987

Hao Bai-lin

Centre of Theoretical Physics

CCAST (World Laboratory)

and

Institute of Theoretical Physics

Academia Sinica, Beijing

PR China 


\section{CONTENTS}

Foreword $\ldots \ldots \ldots \ldots \ldots \ldots \ldots \ldots \ldots \ldots \ldots \ldots \ldots$

DIRECTIONS IN CLASSICAL CHAOS 1

Joseph Ford

1. Back to the Future $\ldots \ldots \ldots \ldots \ldots \ldots \ldots \ldots \ldots \ldots$

2. Generalized Uncertainty Principle $\ldots \ldots \ldots \ldots \ldots \ldots \ldots \ldots \ldots \ldots$

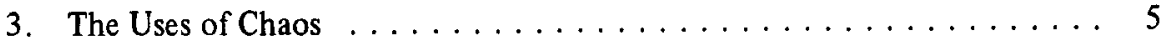

4. Algorithmic Integrability $\ldots \ldots \ldots \ldots \ldots \ldots \ldots \ldots \ldots \ldots$

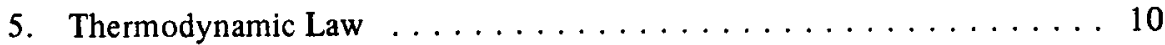

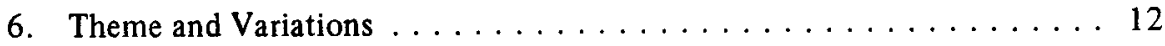

NONLINEAR RESONANCE AND CHAOS IN CONSERVATIVE SYSTEMS

L. E. Reichl \& W. M. Zheng

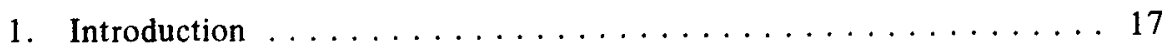

2. Important Properties of Hamiltonian Systems $\ldots \ldots \ldots \ldots \ldots \ldots \ldots 21$

3. Unperturbed Double Well and Pendulum Systems . . . . . . . . 26

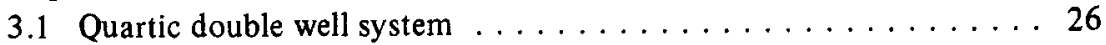

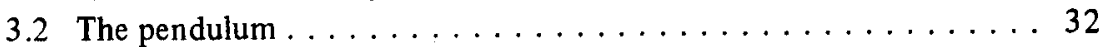

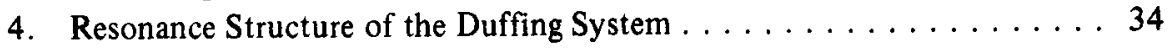

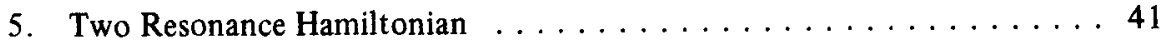

6. Behavior near the Separatrix - Melnikov Integral . . . . . . . . 51

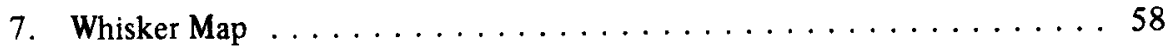

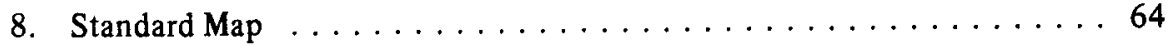

9. Cantori .......................... 73

Appendix A: Elliptic Functions and Integrals . . . . . . . . . 79

Appendix B: Renormalization Group Transformation ..........81

Appendix $C$ : Evaluation of Melnikov Integral $\ldots \ldots \ldots \ldots \ldots \ldots$ 


\section{GENERALIZED RENORMALIZATION GROUP ANALYSIS OF PERIOD.DOUBLING BIFURCATIONS}

K. L. Liu \& K. Young

1. Introduction $\ldots \ldots \ldots \ldots \ldots \ldots \ldots \ldots \ldots \ldots \ldots \ldots \ldots \ldots \ldots$

2. Renormalization Group . . . . . . . . . . . . . . 94

3. Truncation $\ldots \ldots \ldots \ldots \ldots \ldots \ldots \ldots \ldots \ldots \ldots \ldots$

3.1 Analytic solution of $(3,3)$ order . . . . . . . . . . . 97

3.2 Results for higher orders . . . . . . . . . . . . . . 99

4. Functional Relations . . . . . . . . . . . . . . . . . . 101

4.1 Functional derivation . . . . . . . . . . . . . . . 101

4.2 "Experimental" derivation $\ldots \ldots \ldots \ldots \ldots \ldots \ldots \ldots \ldots \ldots$

4.3 Discussion ....................... 105

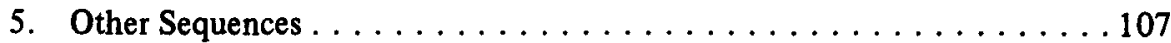

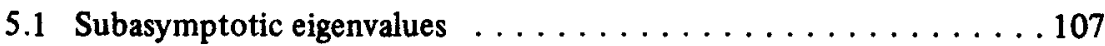

5.2 Generalized RG . . . . . . . . . . . . . . . . 108

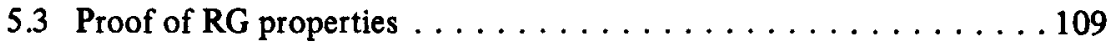

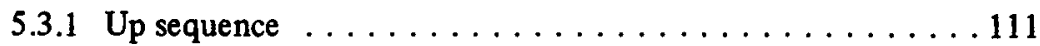

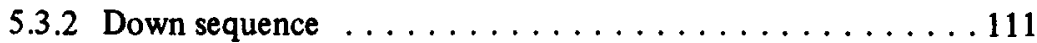

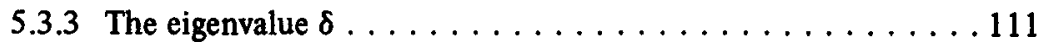

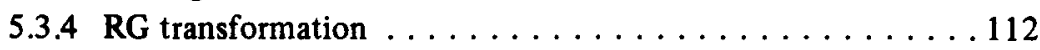

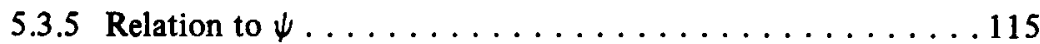

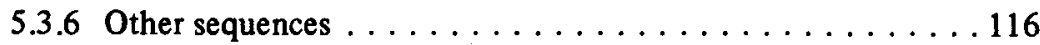

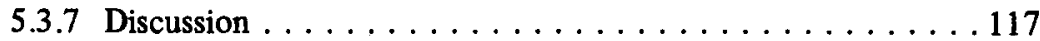

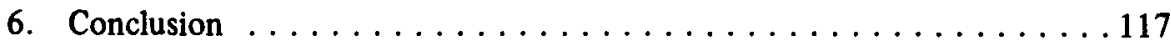

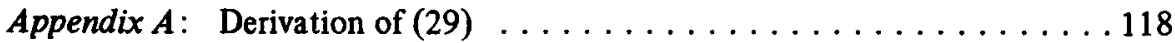

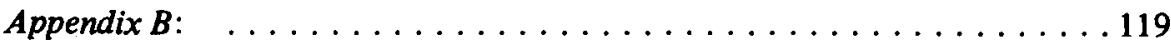

APPLICATION OF DIMENSION ALGORITHMS TO EXPERIMENTAL CHAOS

Gottfried Mayer-Kress

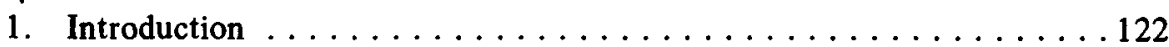

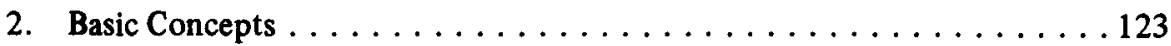

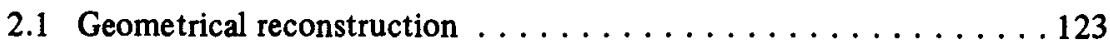

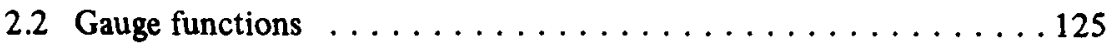

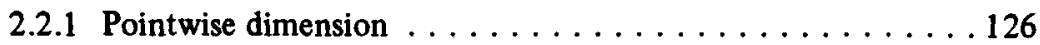

2.2.2 The "correlation-" or "Grassberger-Procaccia" dimension . . . . . . . . . . . . . . . . 127 
2.2.3 Information-(mass) dimension $\ldots \ldots \ldots \ldots \ldots \ldots \ldots 129$

2.2.4 Averaged pointwise dimension . . . . . . . . . 129

2.2.5 Average distances of nearest neighbors . . . . . . . 130

3. Reliability and Stability of the Algorithms $\ldots \ldots \ldots \ldots \ldots 131$

3.1 Minimal number of data points and sampling rate of

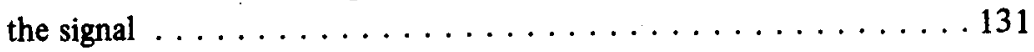

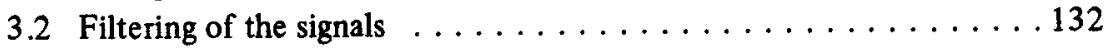

3.3 Geometrical effects . . . . . . . . . . . . . . 134

3.4 Random walks . . . . . . . . . . . . . 135

\section{EMERGENCE OF CHAOS IN LASER SYSTEMS AND}

THE DEVELOPMENT OF DIAGNOSTIC TECHNIQUES

J. R. Tredicce \& L.M. Narducci

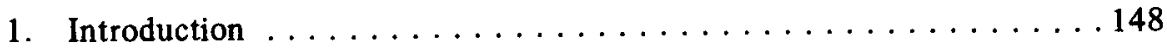

2. Classification of Lasers $\ldots \ldots \ldots \ldots \ldots \ldots \ldots \ldots \ldots \ldots \ldots$

3. Chaotic Laser Systems . . . . . . . . . . . . . . . . . 151

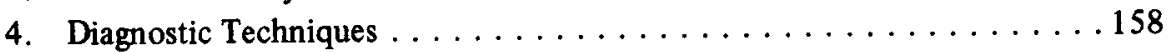

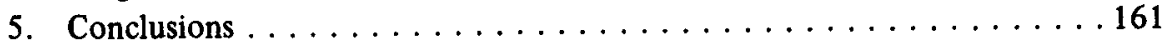

DISSIPATIVE CLASSICAL AND QUANTUM DYNAMICS: THE MORSE OSCILLATOR

Jian-Min Yuan

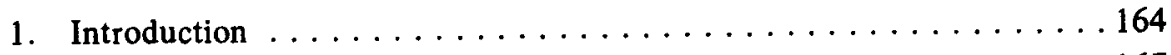

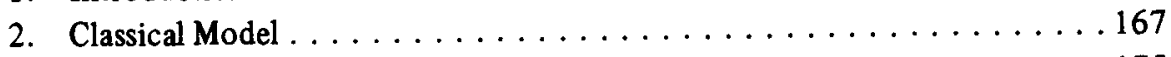

3. Semiclassical Model . . . . . . . . . . . . . . . . 175

4. Quantum Mechanical Study . . . . . . . . . . . . . 184

5. Experimental Verification of Bistable and Chaotic Behavior . . . . . 196

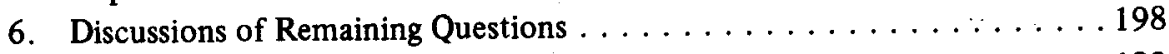

7. Summary . . . . . . . . . . . . . . . . . 199

TRANSITIONS TO CHAOS IN HIGHER DIMENSIONS 206 Bambi Hu \& Jian-Min Mao

1. Introduction . . . . . . . . . . . . . . . . 206

2. Period Doubling in Four-Dimensional

Volume-Preserving Maps. 
2.1 Introduction . . . . . . . . . . . . . . . . . . 208

2.1.1 Canonical transformation and symplecticity $\ldots \ldots \ldots 208$

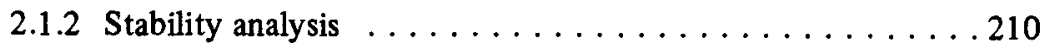

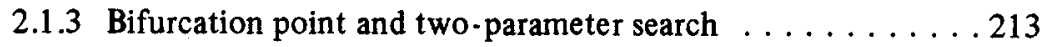

2.2 Search for a complete period-doubling sequence $\ldots \ldots \ldots \ldots 215$

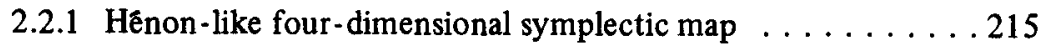

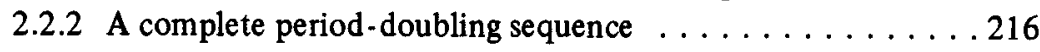

2.2 .3 Scaling factors . . . . . . . . . . . . . . . . 218

2.3 Further search for other period-doubling sequences in

symmetric four-dimensional volume-preserving maps . . . . . . 225

2.3.1 Bifurcation of stability regions in the parameter plane . . . 226

2.3.2 Period-doubling bifurcation routes and scaling factors . . . . 230

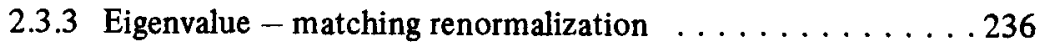

2.4 Fixed maps of the renormalization operator . . . . . . . . 239

2.4.1 Symmetric four-dimensional volume-preserving maps . . . . . 239

2.4.2 Renormalization calculation . . . . . . . . . . . . 243

2.4.3 Three fixed maps corresponding to pitchfork bifurcation . . . . . . . . . . . . . . . 244

2.4.4 Renormalization of asymmetric volume-preserving four-dimensional maps . . . . . . . . . . . . . 248

2.5 Summary . . . . . . . . . . . . . . . . . . . . 252

3. Quasiperiodicity with Three Incommensurate Frequencies . . . . . . 253

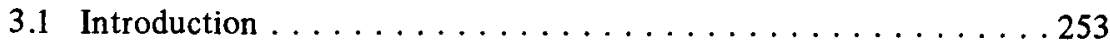

3.2 Formulation of the three-frequency problem $\ldots \ldots \ldots \ldots 254$

3.2.1 Review of the two-frequency problem . . . . . . . 254

3.2.2 Generalization to three frequencies . . . . . . . . 258

3.3 Singular homeomorphisms on torus . . . . . . . . . 262

3.4 Numerical results . . . . . . . . . . . . . . . . . 263

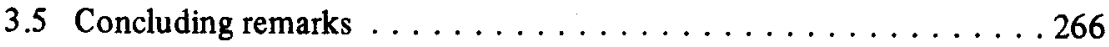

PHENOMENOLOGY OF SPATIO-TEMPORAL CHAOS 272 James $P$. Crutchfield \& Kunihiko Kaneko

1. Complexity and Statistical Mechanics of Deterministic Behavior . . . . 272

2. Lattice Dynamical Systems: Prototypes for Spatio-Temporal

Complexity . . . . . . . . . . . . . . . . . . . . . . 276

2.1 Restriction to spatial lattices . . . . . . . . . . . . 276

2.2 Architectural relationship to other spatially-extended systems . . . . . . . . . . . . . . . . . . . 278 
2.3 Choice of local dynamics . . . . . . . . . . . . . 280

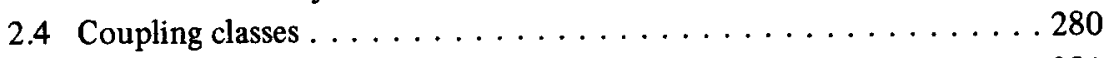

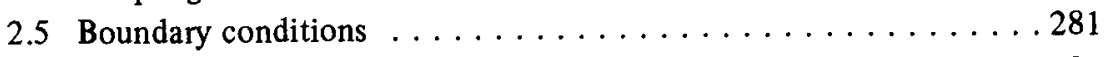

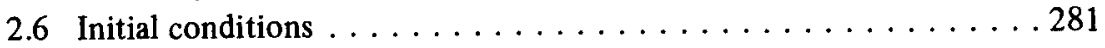

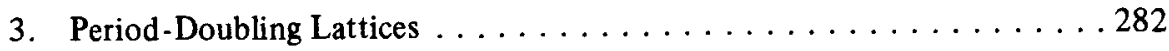

3.1 Dependence of domain behavior on domain size $\ldots \ldots \ldots \ldots 287$

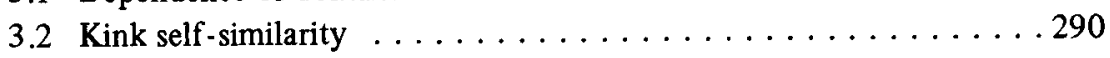

4. Spatial Mode Instability: The Transition from Torus to Chaos . . . . . 290

5. Soliton Turbulence . . . . . . . . . . . . . . . . 313

6. Intermittency Lattices . . . . . . . . . . . . . 317

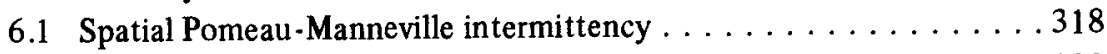

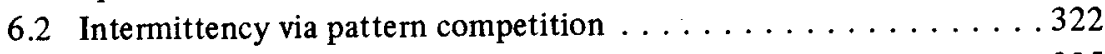

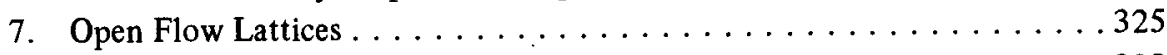

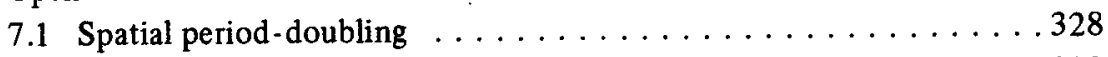

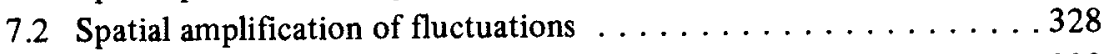

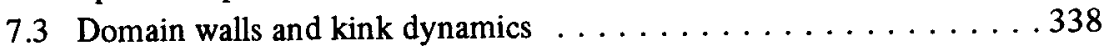

8. Transient Spatial Chaos . . . . . . . . . . . . . . . . . 339

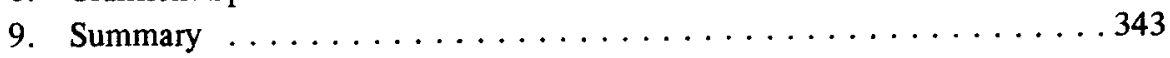

